\title{
Comparison of local and global approaches to digital image
} correlation

\author{
François Hild $^{\star}$. Stéphane Roux
}

Received: date / Accepted: date

\begin{abstract}
Local and global approaches to digital image correlation are compared when the displacement interpolation is based upon bilinear shape functions (i.e., with fournode quadrilaterals). The resolution in terms of displacements and strains associated with both techniques are evaluated a priori and validated a posteriori by using series of images of real experiments. It is shown that global approaches generally out-perform a local approach.
\end{abstract}

Keywords 4-noded quadrilaterals · Displacement · Full-field measurements ·

Resolution $\cdot$ Standard uncertainty $\cdot$ Strain.

\section{Introduction}

Digital image correlation (DIC) is a popular technique to measure 2D and 3D displacement fields [1-3]. In its 2D version, DIC consists in registering two images by measuring the displacement field that enables for the best match. Generally, planar surfaces normal to the optical axis are observed to minimize perspective distortions, and non-rigid image registration is sought for. A random pattern is often applied on the observed Laboratoire de Mécanique et Technologie (LMT-Cachan) ENS Cachan / CNRS / UPMC / PRES UniverSud Paris 61 Avenue du Président Wilson, F-94235 Cachan Cedex, France

${ }^{\star}$ Corresponding author: hild@lmt.ens-cachan.fr 
surface, or the natural surface texture and markings are exploited to measure the displacement field based on the assumption that the surface texture passively follows the displacement of the analyzed solid. DIC, which is used in solid mechanics, is equivalent to Particle Image Velocimetry (PIV) utilized in fluid mechanics. DIC [4-7] was initiated a few years later than PIV [8-12]. However, because the sought displacement and strain resolutions were smaller than the velocity resolutions, its development was slower; algorithmic challenges being more difficult to address. However, the technique has reached centipixel standard resolutions and uncertainties [13-16], and millipixel biases or less $[17,14,2,16]$ that are compatible with a large variety of cases encountered in solid mechanics [1-3].

In its original developments, the registration was performed on zones of interest (ZOIs) that are small windows of the considered region of interest. This type of approach will be referred to as local. The early developments were based on pure translations that were sought either in the physical space [4-6] or in Fourier space [18-20]. The kinematic hypotheses were changed later on to account for warping of the ZOI and first order interpolations were implemented [7,21]. Higher degree interpolations are also used [15] but in all the cases, the only information that is kept is the mean displacement of each ZOI.

In computer vision, very early on, alternative techniques were implemented. They are based upon variational formulations (as one would use in solid mechanics when resorting to, say, finite element formulations [22]). In the work of Horn and Schunck, a spatial regularization was introduced to reduce the displacement fluctuations [23]. However, in cases in which discontinuities are expected, the previous approach is not appropriate per se [24]. "Smoother" penalizations based on robust statistics were implemented $[25,26]$. Another way of regularizing the registration is to enforce directly, say, continuity or even more so that no choice is left concerning the weighting associated with the regularization term. Various displacements fields were studied, namely, linear [27], Fourier expansions [27, 28], B-spline [29,30], elastic solutions (closed-form [31, 
$32]$ and numerical $[33,34])$. In all these cases, because the registration is performed over the whole region of interest (ROI), it will be referred to as global approach.

It is also possible to use the same interpolations as those used in local approaches. In that case, there is a need for discretization schemes and the finite element method is the most suited one. In computer vision, this type of approach was developed in the 1990s [35]. Finite-element based global approaches were introduced in the mid2000s in solid mechanics. Either the correlation product [37] or the sum of squared differences $[36,14]$ were used as the minimization quantity. 4-noded bilinear elements are the most logical choice for comparison purposes. They will be used herein in addition to mesh adaption to allow for better resolutions of nodal displacements in the whole ROI. It is worth noting that unstructured meshes could also be used [33]. However, there are very few, if any, local approaches that use such types of discretizations.

The aim of the paper is to compare local and global approaches. For local as for global approaches, different matching algorithms are used [15,36,37,14]. To make the comparison objective, the same algorithms should be used in terms of gray level interpolation, kinematic interpolation, and minimization procedure. It will be the case hereafter. The paper is organized as follows. First, the principles of local and global approaches will be presented when the sum of squared differences is minimized to evaluate the sought displacements. The sensitivity to acquisition noise is discussed in terms of the uncertainty levels of nodal displacements, mean displacements and mean strains per ZOI or element. Theoretical predictions are compared with an a priori resolution analysis. A first practical example deals with a series of pictures taken with a CCD camera during a biaxial test on a thin film. A couple of pictures acquired in a scanning electron microscope (SEM) are analyzed as a second example. 


\section{Local and global approaches to DIC}

\subsection{Correlation procedure}

There are various ways of registering $2 \mathrm{D}$ pictures. Among them, the most popular in local approaches is related to the correlation product [2]. An alternative approach is given by the sum of squared differences. The latter was used by the authors when implementing global approaches in the context of finite element discretizations [14, 32-34]. It will be used for both techniques in the sequel.

Let us consider $f$ the picture of the reference configuration, and $g$ that in the deformed configuration. Those two images capture the speckle pattern of the solid surface that is assumed to be passively advected by the supporting solid. The gray level conservation at any pixel location $\mathbf{x}$ then reads

$$
f(\mathbf{x})=g(\mathbf{x}+\mathbf{u}(\mathbf{x}))
$$

where $\mathbf{u}$ is the displacement vector. Because the conservation law is never strictly satisfied due to acquisition noise and the number of unknowns exceed the number of equations, it is not possible to determine the displacement vector $\mathbf{u}$ from the sole pictures $f$ and $g$. The correlation procedure has therefore to be written on a given domain that contains more than 1 pixel. Thus a correlation procedure aims, for instance, at minimizing the sum of squared differences

$$
\mathcal{T}=\int_{\Omega}(f(\mathbf{x})-g[\mathbf{x}+\mathbf{u}(\mathbf{x})])^{2} \mathrm{~d} \mathbf{x}
$$

over the considered domain $\Omega$ (i.e., a ZOI in a local approach, and a ROI in a global approach) in which the displacement field is interpolated as

$$
\mathbf{u}(\mathbf{x})=\sum_{n} u_{n} \psi_{n}(\mathbf{x})
$$

where $\boldsymbol{\psi}_{n}$ are (chosen) vector functions, and $u_{n}$ the associated degrees of freedom. The measurement problem then consists in minimizing $\mathcal{T}$ with respect to the unknowns $u_{n}$. Let us note that choosing nodal displacements is a convenient way to emphasize the 
similarities and differences between local and global approaches. However, the traditional parametrization used in local approaches is equivalent but different. The center point displacement being the key quantity of interest it is singled out, and other degrees of freedom correspond to a first order polynomial expansion about the center.

A Newton iterative procedure is followed to circumvent the nonlinear aspect of the minimization problem. Let $\mathbf{u}^{i}$ denote the displacement at iteration $i$, and $\{\mathbf{u}\}^{i}$ the vector containing all the unknown degrees of freedom. By assuming small increments $\mathrm{d} \mathbf{u}=\mathbf{u}^{i+1}-\mathbf{u}^{i}$ of the solution, a Taylor expansion is used to linearize $g(\mathbf{x}+\mathbf{v}(\mathbf{x})) \approx$ $g(\mathbf{x})+\mathbf{v}(\mathbf{x}) \cdot \boldsymbol{\nabla} g(\mathbf{x}) \approx g(\mathbf{x})+\mathbf{v}(\mathbf{x}) \cdot \boldsymbol{\nabla} f(\mathbf{x})$ and then, $\partial \mathcal{T} / \partial\{\mathbf{u}\}^{i}$ is recast in a matrixvector product as

$$
\frac{\partial \mathcal{T}}{\partial\{\mathbf{u}\}^{i}}=[\mathbf{M}]\{\mathrm{d} \mathbf{u}\}-\{\mathbf{b}\}^{i}=\{\mathbf{0}\}
$$

with

$$
\begin{aligned}
M_{m n} & =\int_{\Omega}\left[\boldsymbol{\nabla} f(\mathbf{x}) \cdot \boldsymbol{\psi}_{m}(\mathbf{x})\right]\left[\boldsymbol{\nabla} f(\mathbf{x}) \cdot \boldsymbol{\psi}_{n}(\mathbf{x})\right] \mathrm{d} \mathbf{x} \\
b_{m}^{i} & =\int_{\Omega}\left[f(\mathbf{x})-\tilde{g}^{i}(\mathbf{x})\right]\left[\boldsymbol{\nabla} f(\mathbf{x}) \cdot \boldsymbol{\psi}_{m}(\mathbf{x})\right] \mathrm{d} \mathbf{x}
\end{aligned}
$$

and

$$
\tilde{g}^{i}(\mathbf{x})=g\left(\mathbf{x}+\mathbf{u}^{i}(\mathbf{x})\right)
$$

It is worth noting that matrix $[\mathbf{M}]$ is computed once for all, and only vector $\{\mathbf{b}\}$ is updated from one iteration to the next. This choice (other alternatives are possible [14]) is only dictated by computational efficiency. The iterations stop when the displacement corrections $\{\mathrm{d} \mathbf{u}\}$ reach a small level that is chosen by the user. In many situations, the initial value is $\{\mathbf{u}\}^{i}=\{\mathbf{0}\}$, but there are other propositions [30,38].

To evaluate the resolution ${ }^{1}$ of the developed technique, since the un-noised reference is not known, the noise is assigned to the deformed picture with a variance $2 \sigma^{2}$, where $\sigma^{2}$ is the variance of Gaussian white noise [32]. Under this assumption, the covariance matrix $[\mathbf{C}]$ simply reduces to $[14,32]$

$$
[\mathbf{C}]=2 \sigma^{2} p^{2}[\mathbf{K}]
$$

1 The resolution of a measuring system is the 'smallest change in a quantity being measured that causes a perceptible change in the corresponding indication' [39]. 
with $[\mathbf{K}]=[\mathbf{M}]^{-1}$, and $p$ the physical size of one pixel. This result is very general. Similarly, it can be shown that when the noise level remains small, the estimate of $\mathbf{u}$ is unbiased.

\subsection{Local approach}

In the present setting, a local approach to DIC consists in minimizing the sum of squared differences

$$
\mathcal{T}_{l}=\int_{\text {ZOI }}(f(\mathbf{x})-g[\mathbf{x}+\mathbf{u}(\mathbf{x})])^{2} \mathrm{~d} \mathbf{x}
$$

over the considered ZOI. At this level of generality, many choices can be made for the displacement in each ZOI. In the following, shape functions used in finite element formulations are chosen. It is worth noting that the connectivity between elements is not yet enforced in this part. Since pictures are sampled in pixels it is logical to use square or rectangular ZOIs. Only regular square ZOIs will be considered herein. Their size is denoted by $\ell$. The separation between ZOIs can be chosen, namely, it can be equal to $\ell$ (i.e., contiguous ZOIs), less than $\ell$ (i.e., overlapping ZOIs), or greater than $\ell$ (i.e., separate ZOIs). To compare with finite element calculations, contiguous ZOIs will be used in the sequel. The simplest interpolation to consider is bilinear (i.e., $1, x, y, x y$ functions, where $x$ and $y$ denote the local coordinates of any point $M(x, y)$ belonging to a given ZOI). Each component of the displacement field is treated the same way (i.e., only scalar functions $N_{n}(\mathbf{x})$ are considered) so that the displacement $\mathbf{u}^{e}(\mathbf{x})$ in each ZOI $\Omega_{e}$ reads

$$
\mathbf{u}^{e}(\mathbf{x})=\sum_{n=1}^{n_{e}} \sum_{\alpha} a_{\alpha n}^{e} N_{n}(\mathbf{x}) \mathbf{e}_{\alpha}
$$

where $n_{e}$ denotes the number of nodes (here $\left.n_{e}=4\right), \mathbf{e}_{\alpha}$ the unit vector associated with direction $\alpha=1,2$, and $a_{\alpha n}^{e}$ the unknown nodal displacements. Elementary matrices $\left[\mathbf{M}^{e}\right]$ whose components read

$$
M_{\alpha m \beta n}^{e}=\int_{\Omega_{e}} \partial_{\alpha} f(\mathbf{x}) N_{m}(\mathbf{x}) \partial_{\beta} f(\mathbf{x}) N_{n}(\mathbf{x}) \mathrm{d} \mathbf{x}
$$

and elementary vectors $\left\{\mathbf{b}^{e}\right\}$

$$
b_{\alpha m}^{e}=\int[f(\mathbf{x})-\tilde{g}(\mathbf{x})] \partial_{\alpha} f(\mathbf{x}) N_{m}(\mathbf{x}) \mathrm{d} \mathbf{x}
$$


where $\partial_{\alpha} f=\nabla f \cdot \mathbf{e}_{\alpha}$, and $\tilde{g}$ the corrected image, are computed for each ZOI. Matrix $\left[\mathbf{M}^{e}\right]$ is symmetric, positive, and definite (when invertible). Contrary to classical FE procedures, quadrature formulas are not used because of the irregularity of the picture texture. Instead, a pixel summation is implemented [14]. For each ZOI, an $8 \times 8$ linear system is solved iteratively

$$
\left[\mathbf{M}^{e}\right]\left\{\mathrm{d} \mathbf{a}^{e}\right\}=\left\{\mathbf{b}^{e}\right\}^{i}
$$

where vector $\left\{\mathbf{a}^{e}\right\}$ collects all sought degrees of freedom $a_{\alpha n}^{e}$. The resolution analysis of Section 2.1 is applied to the kinematics chosen in this part. For each degree of freedom $a_{\alpha m}^{e}$, the standard variation $\sigma_{a_{\alpha m}^{e}}$ reads

$$
\sigma_{a_{\alpha m}^{e}}=\sqrt{2} \sigma p \sqrt{K_{\alpha m \alpha m}^{e}}
$$

with $\left[\mathbf{K}^{e}\right]=\left[\mathbf{M}^{e}\right]^{-1}$. This result shows that depending on the underlying texture, and more precisely its gradient, the resolution of the correlation technique is not necessarily uniform over the whole ROI.

Let us assume that the correlation length of the texture is greater than a few pixels and less than the ZOI size $\ell$. In such a case, a "mean-field" assumption is used, namely, the squared image gradient that appears in the expression of $\left[\mathbf{M}^{e}\right]$ (Equation (11)) is extracted from the integral and changed into its expectation value

$$
\left\langle\partial_{\alpha} f \partial_{\beta} f\right\rangle=G_{f}^{2} \delta_{\alpha \beta}
$$

where $G_{f}$ is the square mean of the image gradient projected along any direction. Note that here the image texture is assumed to be isotropic. This mean-field assumption is expected to hold for large ZOI sizes, but it may break down for small ones. Under such circumstances, sub-matrix $\mathbf{M}_{\alpha \beta}^{e}$ reads

$$
\left[\mathbf{M}_{\alpha \beta}^{e}\right] \propto \frac{G_{f}^{2} \ell^{2}}{36} \delta_{\alpha \beta}\left[\begin{array}{cccc}
4 & 2 & 2 & 1 \\
2 & 4 & 1 & 2 \\
2 & 1 & 4 & 2 \\
1 & 2 & 2 & 4
\end{array}\right]
$$


Consequently, the covariance matrix associated with the measured degrees of freedom reads

$$
\left[\mathbf{C}_{\alpha \beta}\right] \propto \frac{8 \sigma^{2} p^{2}}{G_{f}^{2} \ell^{2}} \delta_{\alpha \beta}\left[\begin{array}{cccc}
4 & -2 & -2 & 1 \\
-2 & 4 & 1 & -2 \\
-2 & 1 & 4 & -2 \\
1 & -2 & -2 & 4
\end{array}\right]
$$

The covariance matrix for the degrees of freedom is simply related to the inverse of $[\mathbf{M}]$. Thus there exists a basis where both $\left[\mathbf{M}^{e}\right]$ and $[\mathbf{C}]$ are diagonal. The spectrum of eigenvalues and eigenmodes allows for a simple interpretation of noise sensitivity. In a local approach, the four eigenvalues of $\left[\mathbf{M}_{\alpha \alpha}^{e}\right]$ are $G_{f}^{2} \ell^{2}(1 / 4,1 / 12,1 / 12,1 / 36)$. The largest eigenvalue corresponds to a uniform translation. The second eigenvalue, which is twice degenerate and three times smaller than the first one, corresponds to a uniform gradient along $x$ and $y$. Finally, the fourth eigenvalue is a field whose nodal value is proportional to $\{1,-1,-1,1\}$, and as it is orthogonal to the three other eigenmodes, it corresponds to a deformation mode with zero translation and zero mean strain. However, it is nine times smaller than the major one. Thus noise has a much more pronounced effect on the latter one.

An estimate $\sigma_{a}$ of the standard displacement resolution of each degree of freedom is given by

$$
\sigma_{a}=\frac{4 \sqrt{2} \sigma p}{G_{f} \ell}
$$

For any quantity of interest, which is linear in the displacement $\varphi=\sum_{i} \kappa_{i} a_{i}$, or $\varphi=\{\boldsymbol{\kappa}\}^{t}\{\mathbf{a}\}$, its standard uncertainty reads

$$
\sigma_{\varphi}=\left(\{\boldsymbol{\kappa}\}^{t}[\mathbf{C}]\{\boldsymbol{\kappa}\}\right)^{1 / 2}
$$

where $\kappa_{i}$ are known coefficients collected in vector $\{\boldsymbol{\kappa}\}$. This general formula can be used to evaluate the mean displacement uncertainty, the strain uncertainty, but also holds for more complex linear forms such as stress intensity factors (based on interaction integrals $[40,41])$, or simply mean strain evaluated over arbitrary shaped and sized domains. 
For any point $\mathrm{x}$ within a ZOI, the displacement can be evaluated from the different shape functions, see Equation (10). Consequently, Equation (19) can be used to map out the uncertainty of $\sigma_{u}(\mathbf{x})$. The shape of the uncertainty function has a minimum at the centre and a maximum value at the mesh nodes. The map is shown in Figure 1(a). Consequences of this observation are that if one single information is to be kept from the local analysis then the center value is the most reliable one. The displacement at the center of ZOI reads

$$
\bar{u}=\frac{1}{4} \sum_{m=1,4} a_{\alpha m}^{e}
$$

and the corresponding standard deviation

$$
\sigma_{\bar{u}}=\sqrt{2} \sigma p \sqrt{\sum_{m=1,4} \sum_{n=1,4} K_{\alpha m \alpha n}^{e}}=\frac{\sigma_{a}}{4}
$$

To evaluate the strain field, the components of the displacement gradient $\boldsymbol{\nabla} \otimes \mathbf{u}^{e}$ is computed in each ZOI $\Omega_{e}$ by using the gradient of the shape functions

$$
\left(\nabla \otimes \mathbf{u}^{e}\right)(\mathbf{x})=\sum_{n=1}^{n_{e}} \sum_{\alpha} a_{\alpha n}^{e}\left(\nabla N_{n}(\mathbf{x}) \otimes \mathbf{e}_{\alpha}\right)
$$

The mean component per ZOI is calculated by using the divergence theorem

$$
p^{2} \ell^{2} \overline{\boldsymbol{\nabla} \otimes \mathbf{u}}^{e}=\int_{\partial \Omega_{e}}\left(\mathbf{u}^{e} \otimes \mathbf{n}\right) \mathrm{d} s
$$

where $\mathbf{n}$ is the outward normal of any point belonging to the boundary $\partial \Omega_{e}$ characterized by its curvilinear abscissa $s$. Let $u_{\alpha, \alpha}$ and $u_{\alpha, \beta}$ denote the two components of the displacement gradient associated with $u_{\alpha}$. The two average quantities over any ZOI are related to the degrees of freedom $a_{\alpha m}^{e}$ by

$$
\overline{u_{\alpha, \alpha}}=\frac{a_{\alpha 4}^{e}-a_{\alpha 3}^{e}+a_{\alpha 2}^{e}-a_{\alpha 1}^{e}}{2 \ell p} \quad, \quad \overline{u_{\alpha, \beta}}=\frac{a_{\alpha 4}^{e}+a_{\alpha 3}^{e}-a_{\alpha 2}^{e}-a_{\alpha 1}^{e}}{2 \ell p}
$$

so that their corresponding standard deviations reads

$$
\begin{aligned}
\sigma_{\overline{u_{\alpha, \alpha}}} & =\frac{\sqrt{2} \sigma}{2 \ell} \sqrt{\sum_{m=1,4} \sum_{n=1,4}(-1)^{m+n} M_{m n}^{-1}} \\
\sigma_{\overline{u_{\alpha, \beta}}} & =\frac{\sqrt{2} \sigma}{2 \ell} \sqrt{\sum_{m=1,4} \sum_{n=1,4}(-1)^{m+n} M_{n m}^{-1}}
\end{aligned}
$$


As a first approximation, the standard uncertainty level for the mean gradient components $\sigma_{\bar{\epsilon}}$ becomes

$$
\sigma_{\bar{\epsilon}}=\frac{2 \sqrt{6} \sigma}{G_{f} \ell^{2}}
$$

so that it is related to $\sigma_{\bar{u}}$ and $\sigma_{a}$ by

$$
\sigma_{\bar{\epsilon}}=2 \sqrt{3} \frac{\sigma_{\bar{u}}}{\ell p}=\frac{\sqrt{3}}{2} \frac{\sigma_{a}}{\ell p}
$$

Usually, the mean displacement gradients are evaluated by interpolating the mean displacements of neighboring ZOIs. In the following analysis, a set of $2 \times 2$ ZOIs is considered. The mean displacement gradients become

$$
\left\langle u_{\alpha, \alpha}\right\rangle=\frac{\bar{u}_{\alpha 4}-\bar{u}_{\alpha 3}+\bar{u}_{\alpha 2}-\bar{u}_{\alpha 1}}{2 \ell p} \quad, \quad\left\langle u_{\alpha, \beta}\right\rangle=\frac{\bar{u}_{\alpha 4}+\bar{u}_{\alpha 3}-\bar{u}_{\alpha 2}-\bar{u}_{\alpha 1}}{2 \ell p}
$$

and the corresponding uncertainty level $\sigma_{\langle\epsilon\rangle}$ becomes

$$
\sigma_{\langle\epsilon\rangle}=\frac{\sqrt{2} \sigma}{G_{f} \ell^{2}}=\frac{\sigma_{\bar{u}}}{\ell p}
$$

where

$\langle\bullet\rangle$ denotes averages taken over $2 \times 2$ ZOIs.

All these results will be analyzed by using a real picture in Section 3. Figure 2 summarizes the results when a local approach is used and $10 \times 10$ ZOIs are considered. By construction, the local approach yields displacement and strain uncertainties (i.e., $\left.\sigma_{a}, \sigma_{\bar{u}}, \sigma_{\bar{\epsilon}}\right)$ that are independent of the location when the texture content (i.e., $\left(\partial_{\alpha} f\right)^{2}$ is uniformly distributed over the whole ROI) and provided the ZOIs do not overlap.

\subsection{Global approach}

In a global approach to DIC the sum of squared differences is minimized when defined over the whole ROI

$$
\mathcal{T}_{g}=\int_{\mathrm{ROI}}(f(\mathbf{x})-g[\mathbf{x}+\mathbf{u}(\mathbf{x})])^{2} \mathrm{~d} \mathbf{x}
$$

In the following, the same shape functions as those chosen in the local approach will be considered. A regular square mesh made of 4-noded elements with a bilinear interpolation of the displacements is constructed. The main difference with the previous 
approach is that the continuity of the displacement field between elements is satisfied. Each component of the displacement field satisfies Equation (10). Matrix [M] (see Section 2.1) is assembled by considering elementary matrices $\left[\mathbf{M}^{e}\right]$ whose components are defined in Equation (11) and vector $\{\mathbf{b}\}$ is assembled by calculating elementary vectors $\left\{\mathbf{b}^{e}\right\}$ expressed in Equation (12). Matrix $[\mathbf{M}]$ is symmetric, positive, definite (when invertible), and sparse as in any FE computation. A single global problem is solved iteratively

$$
[\mathbf{M}]\{\mathrm{d} \mathbf{u}\}=\{\mathbf{b}\}^{i}
$$

where vector $\{\mathbf{b}\}^{i}$ is updated thanks to the picture corrections $\tilde{g}$, and vector $\{\mathbf{u}\}$ collects all sought degrees of freedom. Because of connectivities between elements, the maximum component of matrix $[\mathbf{M}]$ is multiplied by 4 for inner nodes when compared with a local approach. Further, edge nodes and corner nodes have smaller values compared with inner nodes since the connectivities are different.

As shown above, the spectrum of eigenvalues and eigenmodes allows for a simple interpretation of noise sensitivity. In a global approach, and with a torus topology (in order to avoid edge effects), matrix $[\mathbf{M}]$ is easily diagonalized. Translational invariance makes it a convolution. It is thus diagonal in Fourier space, and hence all harmonic functions $e^{i \mathbf{k} \cdot \mathbf{x}}$ are eigenmodes, where $\mathbf{k}$ is the wave vector. Matrix $[\mathbf{M}] /\left(G_{f}^{2} \ell^{2}\right)$ extends over second neighbors with three different values for inner nodes, 16/36, 4/36, and 1/36 respectively for diagonal, first and second neighbors.

Eigenvalues, $\xi$, are determined by applying $[\mathbf{M}]$ onto such Fourier modes. They obey

$$
\xi=\frac{\left(G_{f}^{2} \ell^{2}\right)}{9}\left(4+2 \cos \left(k_{x}\right)+2 \cos \left(k_{y}\right)+\cos \left(k_{x}\right) \cos \left(k_{y}\right)\right)
$$

with $k_{x}=n_{x} \pi / L, k_{y}=n_{y} \pi / L$, and $0 \leq n_{x} \leq L 0 \leq n_{y} \leq L$. Figure 3 shows the magnitude of the eigenvalues as a function of the wavevector $\mathbf{k}$. The largest eigenvalue, $\xi_{\max }=G_{f}^{2} \ell^{2}$, corresponds to $\mathbf{k}=\mathbf{0}$. The corresponding eigenmode is thus a uniform translation. As the wavelength decreases, so does the eigenvalue, and for a large system size, a series expansion of $\xi$ as a function of $\mathbf{k}$ provides the following expression

$$
\xi=G_{f}^{2} \ell^{2}\left(1-(1 / 6)|\mathbf{k}|^{2}\right)
$$


where only the modulus of the wavenumber matters. At the other end of the spectrum, the smallest eigenvalue corresponds to a checkerboard nodal field. In that case, $k_{x}=$ $k_{y}=\pi$, and hence

$$
\xi_{\min }=(1 / 9) G_{f}^{2} \ell^{2}
$$

The condition number of matrix $[\mathbf{M}]$ is thus equal to 9 , provided the mean-field assumption is valid. A consequence of this analysis is that when the noise level is large (or the element size small), the nodal displacement field exhibits a checkerboard type component that can easily be seen. Hopefully, those high frequency modes have little impact on estimates of strain or mean displacement.

The resolution analysis of Section 2.1 is applied to the finite element implementation $[32,14]$. Each inner node belongs to four elements so that the shape function associated with a given inner node spans over 4 elements. Consequently, the variance is divided by 4 , and the standard displacement resolution $\sigma_{a}$ is divided by 2 when compared with the value of a single Q4 element. However, this result does not account for the additional requirement of continuity in the displacement field (i.e., the global approach deals with matrix-vector system that is written on a level of the ROI). To estimate the additional gain associated with the continuity constraint, a global matrix is formed by assembling elementary matrices $\mathbf{M}_{\alpha \alpha}$ for, say, a mesh made of $10 \times 10$ Q4 elements (Figure 2). For inner nodes, it is found that

$$
\sigma_{a}=\frac{\sqrt{6} \sigma p}{G_{f} \ell}
$$

The multiplicative factor induced by continuity is therefore equal to $\sqrt{3} / 2$. Edge nodes are shared by two elements so that the standard displacement resolution $\sigma_{a}$ is divided by $\sqrt{2}$ when compared with its value for a single Q4 element. Again, this result does not account for the continuity of the displacement field. When the latter is accounted for, the following result is obtained

$$
\sigma_{a}=\frac{2 \sqrt{3} \sigma p}{G_{f} \ell}
$$

The multiplicative factor due to continuity is equal $\sqrt{3} / 2$ as for the previous case. Last, corner nodes belong to one element only, and the standard displacement resolution $\sigma_{a}$ 
is identical to that of a single Q4 element. By following the same procedure as above, it is found that

$$
\sigma_{a}=\frac{2 \sqrt{6} \sigma p}{G_{f} \ell}
$$

The multiplicative factor is equal to $\sqrt{3} / 2$ in that case as well. All these results show that there is an additional bonus associated with the continuity requirement on the uncertainty level of the nodal displacements. If this effect is neglected, it is shown that the same uncertainty level is reached for a global approach as for a local one, but with a mesh that is two times finer in both directions. This can be understood by comparing the number of unknown degrees a freedom per direction $\mathbf{e}_{\alpha}$, which is equal to $4 N_{x} N_{y}$ when $N_{x} \times N_{y}$ Q4 ZOIs are analyzed in a local approach, and $\left(N_{x}+\right.$ 1) $\left(N_{y}+1\right)$ when $N_{x} \times N_{y}$ Q4 elements are considered in a global approach. The variance level is estimated as the ratio of the total number of pixels in the ROI $L_{x} \times L_{y}$ divided by the number of unknowns. Consequently, the gain in uncertainty level is $\sqrt{\left(N_{x}+1\right)\left(N_{y}+1\right) / 4 N_{x} N_{y}} \approx 1 / 2$ when $N_{x}, N_{y} \gg 1$ when the same Q4 discretization is compared for a local and a global approach.

Because the number of connectivities of each node of the mesh is different (i.e., from 1 to 4 ), it leads to a standard uncertainty $\sigma_{a}$ that is no longer uniform over the whole ROI. This is also the case of $\sigma_{\bar{u}}$ and $\sigma_{\bar{\epsilon}}$ (Figure 2). For inner nodes, by using the same approach as above, the following approximations are obtained for $\sigma_{\bar{u}}$

$$
\sigma_{\bar{u}}=\frac{0.63 \sqrt{2} \sigma p}{G_{f} \ell}
$$

and $\sigma_{\bar{\epsilon}}$

$$
\sigma_{\bar{\epsilon}}=\frac{1.67 \sqrt{2} \sigma}{G_{f} \ell^{2}}
$$

In both cases, when normalized by the corresponding value or $\sigma_{a}$, they lead to upper bounds.

In the case of $\sigma_{\bar{u}}$, it can be noted that the factor 2 when compared with a local approach is not found. An upper bound to the ratio is equal to 0.36 (it is reached for inner nodes). This effect can be understood by comparing the uncertainty maps of $\sigma_{u}(\mathbf{x})$ for both approaches (Figure 1). The shape of the uncertainty map is similar 
with a minimum at the center and a maximum value at the mesh nodes. However, it is observed that consistently with the value reported for the nodal and center values, the entire function for the global case lies below that of the local case. It is also observed that the gain is much more important at the nodes, and much smaller for the average value. However, for the global approach the same result holds albeit it may appear as less trivial. In particular, when the displacement field is to be compared with another such field only at discrete points it is much more advantageous to select the central values rather than the nodal ones, while preserving the same density of points, the uncertainty is reduced by more than a factor of 2 .

Conversely, for $\sigma_{\bar{\epsilon}}$ there is a gain of 0.48 . In that case, the bonus of continuity is equal to $4 \%$. However, let us note that the mean strain is never calculated in such a way for a local approach. To compare with a local approach, the displacement gradients are evaluated by interpolating the nodal displacements $2 \times 2$ elements. The mean displacement gradients become

$$
\begin{aligned}
\left\langle u_{\alpha, \alpha}\right\rangle & =\frac{a_{\alpha 3}^{e}-a_{\alpha 1}^{e}+a_{\alpha 6}^{e}-a_{\alpha 4}^{e}+a_{\alpha 9}^{e}-a_{\alpha 7}^{e}}{6 \ell p} \\
\left\langle u_{\alpha, \beta}\right\rangle & =\frac{a_{\alpha 7}^{e}-a_{\alpha 1}^{e}+a_{\alpha 8}^{e}-a_{\alpha 2}^{e}+a_{\alpha 9}^{e}-a_{\alpha 3}^{e}}{6 \ell p}
\end{aligned}
$$

and the corresponding uncertainty level $\sigma_{\langle\epsilon\rangle}$ reads

$$
\sigma_{\langle\epsilon\rangle}=\frac{\sqrt{2} \sigma}{\sqrt{3} G_{f} \ell^{2}}=\frac{\sigma_{a}}{3 \ell p}
$$

for inner nodes. Even though nodal displacements are used, a multiplication factor of $1 / \sqrt{3}$ is obtained when the local and global approaches are compared. Had the mean displacements of each elements been used instead, the result of Equation (29) still applies $\left(\sigma_{\langle\epsilon\rangle}=\sigma_{\bar{u}} / \ell p\right)$, so that

$$
\sigma_{\langle\epsilon\rangle}=\frac{0.63 \sqrt{2} \sigma}{G_{f} \ell^{2}}=\frac{\sigma_{a}}{2.75 \ell p}
$$

which is about $8 \%$ higher compared to the previous case.

The use of a uniform mesh leads to a heterogeneous distribution of standard displacement and strain uncertainties due to the heterogeneity of matrix [M] (see Figure 2). It is possible to make the distribution more uniform. Depending on the type 
of studied quantity, the result will be different. In the following analysis, the standard uncertainty of the nodal displacements is chosen. As a first approximation, it was shown that $\sigma_{a}$ was 2 times larger for corner nodes and $\sqrt{2}$ larger for edge nodes when compared to the value of inner nodes. Since the standard displacement uncertainty is inversely proportional to the element size, it suffices to multiply the size of the corner elements by a factor of 2 , and edge elements then become rectangular. Figure 2 shows the effect on the global matrix for which corner and edge nodes have components that have the same order of magnitude than inner nodes.

Under this assumption, the fluctuation of the $G_{f} \ell \sigma_{a} / \sqrt{2} \sigma$ field varies between 1.73 $(\approx \sqrt{3})$ and 1.82 (Figure 2). The field of $G_{f} \ell \sigma_{\bar{u}} / \sqrt{2} \sigma$ then varies between 0.87 and 0.63 , and the field $G_{f} \ell^{2} \sigma_{\bar{\epsilon}} / \sqrt{2} \sigma$ between 0.7 and 1.67. These fluctuations are smaller than those observed with a regular mesh.

\section{Analysis of a synthetic case}

In this first analysis, an a priori resolution analysis is performed. It consists in considering a given picture $f$ and adding Gaussian white noise with different standard deviations to form picture $g$. These two pictures are correlated by DIC. In a first part, a local analysis with Q4 ZOIs is considered. The aim of the study is to evaluate the measurement uncertainties associated with the measured degrees of freedom, the average displacement per ZOI. The same analysis is run for the first displacement gradients.

Figure 4(a) shows the considered reference picture of the random speckle pattern. It was obtained by spraying a black and white paint. It was used to identify damage parameters by using a biaxial test on a vinylester matrix reinforced by a mat of Eglass fibers $[42,43]$. In the present case, a $512 \times 512$-pixel definition is considered. The histogram (Figure 4(b)) does not cover completely the whole 8-bit dynamic range. The correlation radius of the texture is of the order of 3 pixels. Values of $\sigma$ ranging from 1 to 32 gray levels are considered in the sequel. In many practical situations, $\sigma$ is of the order of 1 percent of the dynamic range for CCD or CMOS cameras. However, other imaging systems may be used (e.g., scanning electron microscopes or atomic 
force microscopes) for which the noise level is significantly larger than that observed for conventional cameras (see Section 5).

The first output of a correlation code to be checked is the residual field $\eta(\mathbf{x})=$ $f(\mathbf{x})-g[\mathbf{x}+\mathbf{u}(\mathbf{x})]$ and its RMS $\bar{\eta}$ over the whole ROI. In the present case, the ZOI separation is equal to the ZOI size so that this measure counts only once each pixel belonging the ROI. Figure 5 shows the change of the RMS residual $\bar{\eta}$ normalized by the dynamic range $\Delta f=\max _{\mathrm{ROI}} f-\min _{\mathrm{ROI}} f$ as a function of the dimensionless noise level $\sqrt{2} \sigma / \Delta f$. A linear trend is shown with a slope of 1 . When the noise level reaches very high values, there is a deviation as the number of ZOIs increases, thereby indicating a higher sensitivity to noise, which is to be expected. Otherwise, there is an almost 1 to 1 correspondence between the two quantities. The fact that the correlation residual is virtually identical to the noise level is an indication that the registration was successful. Furthermore, when the noise level is unknown, as will be shown below, it is a simple way of assessing it by acquiring a series of pictures, performing correlations to account for slight kinematic deviations, and assessing the RMS value of the residual, which is equal to $\sqrt{2} \sigma$ as a first approximation.

The result of Equation (18) is analyzed next. Figure 6(a) shows the change of $\sigma_{a}$ with the ZOI size $\ell$ and for different values of $\sigma$. The uncertainty level is increasing as $\sigma$ increases and $\ell$ decreases. This trend is described by Equation (18). On a more quantitative way, when $\sigma_{a}$ is divided by the noise level $\sigma$, it should fall onto a single curve that varies as $1 / \ell$. The results of Figure $6(\mathrm{~b})$ show that this dependence is captured. A deviation is observed when only one ZOI is considered (i.e., $\ell=512$ pixels) or for very small ZOIs. For large ZOIs, very few points are available, making the statistical content poor. Conversely, for small ZOI sizes, the mean field approximation is less likely to apply as the ZOI size becomes closer to the correlation length of the texture. On a quantitative way, it is found that $\ell \sigma_{a} \Delta f / \sqrt{2} \sigma p \approx 47$ to be compared with $4 \Delta f / G_{f}=50$. This last result validates Equation (18) since $\sigma_{a} \approx 4 \sqrt{2} \sigma p / G_{f} \ell$.

When the mean displacement per ZOI is computed, it is expected that its uncertainty level $\sigma_{\bar{u}}$ decreases when compared with that of the measured degrees of freedom 
$\sigma_{a}$ (see Equation (21)). Figure 7 shows a plot of the ratio $\sigma_{\bar{u}} / \sigma_{a}$ as a function of $\sigma_{a}$. The average value of the ratio is found to be equal to 0.24 (to be compared with the theoretical value of $1 / 4)$. The different data are scattered because, for small ZOIs in particular, there are fluctuations of the mean picture gradient, and the hypothesis of scale separation is less satisfied.

Equation (26) is now studied. The change of $\sigma_{\bar{\epsilon}}$ with the ZOI size $\ell$ and for different values of $\sigma$ is shown in Figure 8(a). The uncertainty level again increases with $\sigma$ and decreases with $\ell$ as expected from Equation (26). When $\sigma_{\bar{\epsilon}}$ is normalized by the noise level $\sigma$ it falls onto a single curve that varies as $1 / \ell^{2}$ (see Figure $8(\mathrm{~b})$ ). A deviation is also present when only one ZOI is considered for the same reason as above. The interpolation of the previous results leads to $\ell^{2} \sigma_{\bar{\epsilon}} \Delta f / \sqrt{2} \sigma \approx 39$ to be compared with $2 \sqrt{3} \Delta f / G_{f}=43$. The value $2 \sqrt{3}$ is therefore a very good estimate of the actual ratio $\sigma_{\bar{\epsilon}} / \sqrt{2} \sigma \ell^{2} G_{f}$.

When the mean strain per ZOI is computed, it is expected that its uncertainty level $\sigma_{\bar{\epsilon}}$ depends linearly on the standard uncertainty of the measured degrees of freedom $\sigma_{a}$ (see Equation (27)) and is inversely proportional to $\ell$. Figure 9 shows a plot of the ratio $\sigma_{\bar{\epsilon}} / \sigma_{a}$ as a function of $\sigma_{a}$. The average value of the ratio is found to be equal to 0.84 , which is very close to the theoretical value $(\sqrt{3} / 2 \approx 0.87)$. All the results found in the present section validate the closed-form solutions derived in Section 2.2.

The performances of a global approach is compared with a local one in Figure 10 when the mean values of the standard uncertainties are considered for all the noise levels. As the ZOI or element sizes decrease, the standard uncertainties $\sigma_{a}, \sigma_{\bar{u}}$ and $\sigma_{\bar{\epsilon}}$ of the global approach tend to the theoretical limits when compared with the values of the local approach. This is due to the fact that for small elements, the proportion of inner nodes becomes dominant. These results validate the a priori predictions of the standard resolution in terms of nodal displacement, mean displacement and mean strain per ZOI or element for local and global approaches. 
4 Analysis of a series of pictures of a biaxial experiment

The series of pictures analyzed in this section were taken before starting a biaxial test on supported thin W films. The sample was positioned in the goniometer center of the DiffAbs experimental station at SOLEIL synchrotron source [44]. Strains using x-ray diffraction and digital image correlation can be measured in this new setup (Figure 11(a)) The two techniques led to very similar results not only in terms of mean strain levels but also concerning the standard strain uncertainties [45]. The optical microscope is composed of a telecentric lens mounted on a CCD camera (12-bit dynamic range, definition: $1392 \times 1024$ pixels). This setup allows for the minimization of out-of-plane effects since a stereoscopic equipment was not possible to implement in the experimental environment. Figure 11(b) shows the considered reference picture. It is the raw surface with a $512 \times 512$ pixel definition. Even though the quality of the texture seems poor at first sight, the histogram (Figure 11(c)) indicates that most of the 12-bit dynamic range is used with a slight saturation so that image acquisition is close to optimal. The correlation radius of the texture is of the order of 3 pixels and isotropic. Hence, the images are considered as suitable for DIC.

Values of $\sigma / \Delta$ ranging from $1 / 256$ to $1 / 32 \Delta f$ are considered in the sequel. Figure 12 shows the result of the resolution analysis for a local approach. The same trends as those observed in Figure 5 are obtained. The predictions of the a priori resolution analysis will be compared to actual measurements. A series of ten pictures, in addition to the reference one, was acquired. The stepping motors of the testing machine were motionless. This is typical of an analysis that can be performed experimentally to assess the actual resolution of the measuring system [39]. The direct DIC analysis of the 10 pictures provides the values of the RMS residual $\bar{\eta}$ in addition to the measurement uncertainties. In the present case, $\bar{\eta} / \Delta f$ varies between 0.008 and 0.025 (see shaded zone in Figure 12). From this information, it is concluded that $\sigma$ varies between $0.5 \%$ and $1.8 \%$ of the dynamic range of the camera, with an average value of $1.0 \%$. Figure 13 shows the change of the standard displacement and strain uncertainties as a function of the ZOI size. The latter ones are compared with the levels predicted by the resolution 
analysis when using the bounds of $\bar{\eta}$ and the results of Figure 12. A good agreement is obtained, thereby validating the a priori resolution analysis.

Let us now compare the measurement uncertainties of local and global approaches. Lower uncertainty levels are to be expected with a global approach. Figure 14(a) shows the change of the standard displacement uncertainty of the measured degrees of freedom for both approaches. In both cases, as expected, the uncertainties increase when the ZOI or element sizes decrease. All approaches converge toward the same value when only one ZOI or element is considered. When $2 \times 2$ elements are chosen, the two global approaches coincide since no mesh adaption was performed. As the number of elements increases, the effect of mesh adaption is visible and a gain is observed in terms of $\sigma_{a}$ level for the same element size. The gain is even more important when compared with the results of the local approach.

Figure 14(b) shows the change of the standard uncertainty of the average displacement per ZOI (local approach) or element (global approach). Let us note that the mean displacement is generally not an information that is given when using a global approach. Generally, the values that are shown are the nodal displacements. Yet, for comparison purposes, the levels of these values are compared herein. As expected from the a priori analysis, the gain between local and global approaches is less important. However, the global approaches still out-perform the local approach as the ZOI or element sizes decrease.

Figure 14(c) shows the change of the standard uncertainty of the mean strain per ZOI (local approach) or element (global approach). It is worth noting that this information is generally not given as an output of a local approach. However, for comparison purposes it is reported herein. As the number of ZOIs or elements increases, the ratio of 2 between $\sigma_{\bar{\epsilon}}$ of the global approach compared with that of a local approach is found. This is faster when mesh adaption is considered. 


\section{Analysis of SEM pictures}

A couple of pictures taken 30 minutes apart in an SEM is finally analyzed. They have an 8-bit digitization. A ROI of $750 \times 750$ pixels is extracted. This case was chosen because of the high acquisition noise level generally associated with SEM pictures as will be shown hereafter. In the present case, the secondary electron mode is selected with an Everhart-Thornley detector (ETD). Because the sample surface was not textured enough, a grid was deposited by resorting to microphotolithography (Figure 15(a)). This type of texture leads to a bimodal histogram (Figure 15(b)) corresponding to the grids (high gray levels) and the raw surface (low gray levels). Moreover, the whole dynamic range of the detector is not used in the present case, but only $80 \%$.

When the couple of pictures is analyzed, the RMS residual $\bar{\eta} / \Delta f$ is found to be of the order of $7 \%$ for local and global approaches, which is significantly higher than what was observed above. The a priori analysis is run on the reference picture of Figure 15(a) for values of $\sigma / \Delta$ ranging from $1 / 64$ to $1 / 8$. By using the results of Figure 16, it is concluded that the noise level associated with the SEM pictures is significantly higher than for a conventional CCD or CMOS camera. This case is therefore interesting to check whether the theoretical predictions survive when the noise level of pictures becomes higher.

In Figure 17(a) the change of the standard uncertainty of the measured degrees of freedom for the three approaches is shown. All the general trends observed in Figure 14(b) are also present in this difficult case. In particular, the global approaches become even more interesting as they allow for a significant decrease of the uncertainty level.

Figure 17(b) shows the change of the standard uncertainty of the average displacement per ZOI (local approach) or element (global approach) as a function of $\ell$. For very large ZOIs, the local approach out-performs the global approach. This may occur since it was shown that the ratio $\sigma_{\bar{\epsilon}} / \sigma_{a}$ is lower for a local approach (i.e., $\left.1 / 4\right)$ compared with a global approach (i.e., 0.36 at most for inner nodes). However, the trend 
is inverted for smaller values of $\ell$ for which the global approaches out-perform again the local one.

Figure 17(c) shows the change of the standard uncertainty of the mean strain per ZOI or element as a function of $\ell$. In the present case, there is a clear benefit from the global approach. Very early, the ratio of 2 between the global and local approaches is reached. Because of the high noise level, there is an additional bonus in using global approaches.

\section{Summary}

A comparison between local and global approaches to DIC was performed by using 4noded zones of interest (local approach) and 4-noded elements (global approach). The same minimization procedure was used in both approaches so that algorithmic issues are not the cause of differences. In both cases, the sum of squared differences was minimized by using a modified Newton scheme. It was shown that the global approach out-performs the local one thanks to the continuity requirements and the fact that shape functions span over four elements for inner nodes. It is worth noting that the results derived herein with Q4 shape functions can be generalized to 8-noded cubes with trilinear shape functions [46] when performing Digital Volume Correlation [47].

General results concerning the resolution in terms of displacement and strains were derived. Under simplifying assumptions, closed-form expressions were obtained. They were checked against artificial and practical cases, and validated in all the examples analyzed herein. In particular, a mesh adaption strategy was proposed to yield nodal displacement uncertainties that are uniform for a global approach, irrespective of the fact that nodes are located inside the region of interest, or on its edges or at corners.

The gain in terms of measurement resolution allows for finer meshes, namely, a ratio of two of the element size compared with the ZOI size yields, as a first approximation, the same uncertainty level for the displacement and strain quantities investigated herein. This result shows that more complex displacement fields can be captured by a global approach when compared with a local approach. Furthermore, there is an ad- 
ditional bonus induced by the continuity constraint on the displacement field, though less significant (i.e., of the order of $10 \%$ ) as compared with the previous effect. It was shown that in case of very noisy images, this requirement made the displacement measurements more robust with a global approach.

The next advantage of FE-based global approaches is their direct link with numerical simulations. The measured displacement fields are very often used for comparison purposes with numerical simulations. The latter ones are, for instance, used for identification and / or validation purposes of constitutive laws and numerical models. If these issues are addressed, it is desirable to have the same kinematic basis whenever possible. If it is not the case, the measured field can be interpolated by using the shape functions of the underlying discretization, and not some exogenous interpolation that was not used during the measurement stage.

\section{Acknowledgments}

The authors wish to thank the members of the Micromechanical Testing Laboratory at EDF R \& D Les Renardières for kindly providing the two SEM pictures analyzed herein.

\section{References}

1. M. A. Sutton, S. R. McNeill, J. D. Helm and Y. J. Chao, Advances in Two-Dimensional and Three-Dimensional Computer Vision, in: Photomechanics, P. K. Rastogi, eds., (Springer, Berlin (Germany), 2000), Topics in Appl. Phys., 77 323-372.

2. M. A. Sutton, J.-J. Orteu and H. Schreier, Image correlation for shape, motion and deformation measurements: Basic Concepts, Theory and Applications, (Springer, New York, NY (USA), 2009).

3. F. Hild and S. Roux, Digital Image Correlation, in: Optical Methods for Solid Mechanics, E. Hack and P. Rastogi, eds., 2012, in press.

4. B. D. Lucas and T. Kanade, An Iterative Image Registration Technique with an Application to Stereo Vision, Proceedings 1981 DARPA Imaging Understanding Workshop, (1981), 121-130. 
5. P. J. Burt, C. Yen and X. Xu, Local correlation measures for motion analysis: a comparative study, Proceedings IEEE Conf. on Pattern Recognition and Image Processing, (1982), 269274 .

6. M. A. Sutton, W. J. Wolters, W. H. Peters, W. F. Ranson and S. R. McNeill, Determination of Displacements Using an Improved Digital Correlation Method, Im. Vis. Comp. 1 [3] (1983) 133-139.

7. M. A. Sutton, M. Cheng, W. H. Peters, Y. J. Chao and S. R. McNeill, Application of an optimized digital correlation method to planar deformation analysis, Im. Vis. Comp. 4 [3] (1986) 143-150.

8. D. B. Barker and M. E. Fourney, Measuring fluid velocities with speckle patterns, Optics Lett. 1 (1977) 135-137.

9. T. D. Dudderar and P. G. Simpkins, Laser Speckle Photography in a Fluid Medium, Nature 270 (1977) 45-47.

10. R. Grousson and S. Mallick, Study of flow pattern in a fluid by scattered laser light, Appl. Optics 16 (1977) 2334-2336.

11. R. J. Adrian, Scattering particle characteristics and their effect on pulsed laser measurements of fluid flow: speckle velocimetry vs. particle image velocimetry, Appl. Optics $\mathbf{2 3}$ (1984) 1690-1691.

12. C. J. D. Pickering and N. A. Halliwell, Speckle Laser in Fluid Flows: Signal Recovery with Two-Step Processing, Appl. Opt. 23 (1984) 1128-1129.

13. S. Bergonnier, F. Hild and S. Roux, Digital image correlation used for mechanical tests on crimped glass wool samples, J. Strain Analysis 40 [2] (2005) 185-197.

14. G. Besnard, F. Hild and S. Roux, "Finite-element" displacement fields analysis from digital images: Application to Portevin-Le Chatelier bands, Exp. Mech. 46 (2006) 789-803.

15. M. Bornert, F. Brémand, P. Doumalin, J.-C. Dupré, M. Fazzini, M. Grédiac, F. Hild, S. Mistou, J. Molimard, J.-J. Orteu, L. Robert, Y. Surrel, P. Vacher and B. Wattrisse, Assessment of Digital Image Correlation measurement errors: Methodology and results, Exp. Mech. 49 [3] (2009) 353-370.

16. Y. Q. Wang, M. A. Sutton, H. A. Bruck and H. W. Schreier, Quantitative Error Assessment in Pattern Matching: Effects of Intensity Pattern Noise, Interpolation, Strain and Image Contrast on Motion Measurements, Strain 45 (2009) 160-178.

17. H. W. Schreier, J. R. Braasch and M. A. Sutton, Systematic errors in digital image correlation caused by intensity interpolation, Opt. Eng. 39 [11] (2000) 2915-2921.

18. D. J. Chen, F. P. Chiang, Y. S. Tan and H. S. Don, Digital Speckle-Displacement Measurement Using a Complex Spectrum Method, Appl. Opt. 32 (1993) 1839-1849. 
19. Y. Berthaud, J. Scholz and J. Thesing, Méthodes optiques et acoustiques de mesures des caractéristiques mécaniques, Proceedings Colloque national MECAMAT "Mécanismes et mécanique des grandes déformations", 1996), 77-80.

20. F. P. Chiang, Q. Wang and F. Lehman, New Developments in Full-Field Strain Measurements Using Speckles, in: Non-Traditional Methods of Sensing Stress, Strain and Damage in Materials and Structures (ASTM, Philadelphia (USA), 1997), STP 1318 156-169.

21. P. Vacher, S. Dumoulin, F. Morestin and S. Mguil-Touchal, Bidimensional strain measurement using digital images, Proceedings of the Institution of Mechanical Engineers, Part C: Journal of Mechanical Engineering Science 213 [8] (1999) 811-817.

22. O. C. Zienkievicz and R. L. Taylor, The Finite Element Method, (McGraw-Hill, London (UK), 4th edition, 1989).

23. B. K. P. Horn and B. G. Schunck, Determining optical flow, Artificial Intelligence 17 (1981) 185-203.

24. A. Mitiche and P. Bouthemy, Computation and analysis of image motion: A synopsis of current problems and methods, Int. J. Comp. Vision. 19 (1996) 29-55.

25. M. Black, Robust Incremental Optical Flow, (PhD dissertation, Yale University, 1992).

26. J.-M. Odobez and P. Bouthemy, Robust multiresolution estimation of parametric motion models, J. Visual Comm. Image Repres. 6 (1995) 348-365.

27. S. Roux, F. Hild and Y. Berthaud, Correlation Image Velocimetry: A Spectral Approach, Appl. Optics 41 [1] (2002) 108-115.

28. B. Wagne, S. Roux and F. Hild, Spectral Approach to Displacement Evaluation From Image Analysis, Eur. Phys. J. AP 17 (2002) 247-252.

29. P. Cheng, M. A. Sutton, H. W. Schreier and S. R. McNeill, Full-field Speckle Pattern Image Correlation with B-Spline Deformation Function, Exp. Mech. 42 [3] (2002) 344-352.

30. F. Hild, S. Roux, R. Gras, N. Guerrero, M. E. Marante and J. Flórez-López, Displacement Measurement Technique for Beam Kinematics, Opt. Lasers Eng. 47 (2009) 495-503.

31. F. Hild and S. Roux, Digital image correlation: From measurement to identification of elastic properties - A review, Strain 42 (2006) 69-80.

32. S. Roux and F. Hild, Stress intensity factor measurements from digital image correlation: post-processing and integrated approaches, Int. J. Fract. 140 [1-4] (2006) 141-157.

33. H. Leclerc, J.-N. Périé, S. Roux and F. Hild, Integrated digital image correlation for the identification of mechanical properties, in: MIRAGE 2009, A. Gagalowicz and W. Philips, eds., (Springer, Berlin, 2009), LNCS 5496 161-171.

34. J. Réthoré, S. Roux and F. Hild, An extended and integrated digital image correlation technique applied to the analysis fractured samples, Eur. J. Comput. Mech. 18 (2009) 285-306. 
35. E. P. Simoncelli, Bayesian Multi-Scale Differential Optical Flow, in: Handbook of Computer Vision and Applications, B. Jähne, H. Haussecker and P. Geissler, eds., (Academic Press, 1999), 2 297-422.

36. G. B. Broggiato, Adaptive image correlation technique for full-field strain measurement, Proceedings 12th Int. Conf. Exp. Mech., 2004.

37. Y. Sun, J. Pang, C. Wong and F. Su, Finite-element formulation for a digital image correlation method, Appl. Optics 44 [34] (2005) 7357-7363.

38. G. Besnard, J.-M. Lagrange, F. Hild, S. Roux and C. Voltz, Characterization of necking phenomena in high speed experiments by using a single camera, EURASIP J. Im. Video. Proc. 2010 [215956] (2010) 15 p.

39. ISO/IEC guide 99-12:2007, International Vocabulary of Metrology - Basic and General Concepts and Associated Terms, VIM, (International Organization for Standardization, Geneva (Switzerland), 2007).

40. J. Réthoré, S. Roux and F. Hild, Noise-robust Stress Intensity Factor Determination from Kinematic Field Measurements, Eng. Fract. Mech. 75 [13] (2008) 3763-3781.

41. J. Réthoré, S. Roux and F. Hild, Optimal and noise-robust extraction of Fracture Mechanics parameters from kinematic measurements, Eng. Fract. Mech. 78 [9] (2011) 1827-1845.

42. D. Claire, F. Hild and S. Roux, A finite element formulation to identify damage fields: The equilibrium gap method, Int. J. Num. Meth. Engng. 61 [2] (2004) 189-208.

43. S. Roux and F. Hild, Digital Image Mechanical Identification (DIMI), Exp. Mech. 48 [4] (2008) 495-508.

44. G. Geandier, D. Thiaudière, R. N. Randriamazaoro, R. Chiron, S. Djaziri, B. Lamongie, Y. Diot, E. Le Bourhis, P. O. Renault, P. Goudeau, A. Bouaffad, O. Castelnau, D. Faurie and F. Hild, Development of a synchrotron biaxial tensile device for in-situ characterization of thin films mechanical response, Rev. Sci. Instrum. 81 [103903] (2010).

45. S. Djaziri, P.-O. Renault, F. Hild, E. Le Bourhis, P. Goudeau, D. Thiaudière and D. Faurie, Combined synchrotron X-ray and image-correlation analyses of biaxially deformed $\mathrm{W} / \mathrm{Cu}$ nanocomposite thin films on Kapton, J. Appl. Cryst. 44 (2011) 1071-1079.

46. H. Leclerc, J.-N. Périé, F. Hild and S. Roux, Digital Volume Correlation: What are the limits to the spatial resolution?, [submitted for publication] (2012).

47. S. Roux, F. Hild, P. Viot and D. Bernard, Three dimensional image correlation from X-Ray computed tomography of solid foam, Comp. Part A 39 [8] (2008) 1253-1265. 


\section{List of Figures}

1 Map of the standard displacement uncertainty $\sigma_{u}$ (normalized by $\sqrt{2} \sigma p / G_{f} \ell$ ) for local (a) and global (b) approaches. Cuts along the diagonal $x=y$ (c). 29

2 Standard uncertainties in terms of nodal displacements $\left(\sigma_{a}\right)$, mean displacements per ZOI or element $\left(\sigma_{\bar{u}}\right)$, and mean strains per ZOI or element $\left(\sigma_{\bar{\epsilon}}\right)$ for three different DIC approaches. In the present case, 100 ZOIs are considered for the local approach (left), 100 elements are considered for the global approach (middle), and 64 elements for the global and optimized approach (right) . . . . . . . . . . . 30

3 Map of the eigenvalues in Fourier space. . . . . . . . . . . . . . . 31

4 Reference picture for the a priori resolution analysis (a), and corresponding histogram (b). The definition of the picture is equal to 256 kpixels

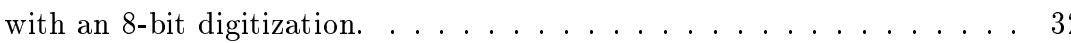

$5 \quad$ Normalized RMS residual $\bar{\eta} / \Delta f$ as a function of the dimensionless noise level $\sqrt{2} \sigma / \Delta f$ when $\sigma=1,2,4,8,16,32$ gray levels. The straight line shows a linear interpolation with a slope of $1 \ldots \ldots . \ldots 33$

6 Standard displacement uncertainty $\sigma_{a}$ as a function of the ZOI size $\ell$ when $\sigma=1,2,4,8,16,32$ gray levels (a). The higher $\sigma$ the higher $\sigma_{a}$ for a given ZOI size. Normalized standard displacement uncertainty $\sigma_{a} \Delta f / \sqrt{2} \sigma$ as a function of the ZOI size $\ell$. The straight line shows an interpolation by the inverse of the ZOI size. . . . . . . . . . . . 34

7 Normalized standard displacement uncertainty $\sigma_{\bar{u}} / \sigma_{a}$ as a function of $\sigma_{a}$. The solid line shows the result of Equation $(21) \ldots \ldots \ldots$

8 Standard strain uncertainty $\sigma_{\bar{\epsilon}}$ as a function of the ZOI size $\ell$ when $\sigma=1,2,4,8,16,32$ gray levels (a). The higher $\sigma$ the higher $\sigma_{\bar{\epsilon}}$ for a given ZOI size. Normalized standard strain uncertainty $\sigma_{\bar{\epsilon}} \Delta f / \sqrt{2} \sigma$ as a function of the ZOI size $\ell$. The solid line shows an interpolation by the inverse of square of the ZOI size. . . . . . . . . . . 36 
9 Normalized standard strain uncertainty $p \ell \sigma_{\bar{\epsilon}} / \sigma_{a}$ as a function of $\sigma_{a}$. The solid line shows the result of Equation $(27) \ldots \ldots . \ldots 37$

10 Standard displacement uncertainty of the measured degrees of freedom (a), of the mean displacement per ZOI (b), of the mean strain per element for a global and optimized approach as functions of the same quantity for a local approach. . . . . . . . . . . . . 38

11 Biaxial testing machine (a) in DiffAbs line at SOLEIL synchrotron [44]. Reference picture of a natural texture used in in situ experiments (b), and corresponding histogram (c). The definition of the picture is equal to 256 kpixels with a 12 -bit digitization. . . . . . . . . . 39

12 Normalized RMS residual $\bar{\eta} / \Delta f$ as a function of the dimensionless noise level $\sqrt{2} \sigma / \Delta f$ when $\sigma / \Delta f=1 / 256,1 / 128,1 / 64,1 / 32$. The solid line shows a linear interpolation with a slope of 1 . The shaded area depicts the variation of correlation residuals for the ten analyzed pictures. . . . 40

13 Standard displacement uncertainty $\sigma_{a}$ (a) and strain uncertainty $\sigma_{\bar{\epsilon}}$ (b) as functions of the ZOI size $\ell$ for the ten analyzed pictures. The gray shaded areas show the predictions based on the a priori resolution analysis. 41

14 Standard displacement uncertainty of the measured degrees of freedom (a), of the mean displacement per ZOI or element (b), of the mean strain per ZOI or element as functions of ZOI or element size $\ell$ for the three approaches used herein. . . . . . . . . . . . . . 42

15 Reference SEM picture for which a grid was deposited (a), and corresponding histogram (b). The definition of the picture is equal to 563 kpixels with an 8 -bit digitization. . . . . . . . . . . . 43

16 Normalized RMS residual $\bar{\eta} / \Delta f$ as a function of the dimensionless noise level $\sqrt{2} \sigma / \Delta f$ when $\sigma / \Delta f=1 / 64,1 / 32,1 / 16,1 / 8$. The solid line shows a linear interpolation with a slope of $1 \ldots \ldots \ldots$. . . . . . . . 44 
17 Standard displacement uncertainty of the measured degrees of freedom (a), of the mean displacement per ZOI or element (b), of the mean strain per ZOI or element as functions of ZOI or element size $\ell$ for the three approaches used herein. 

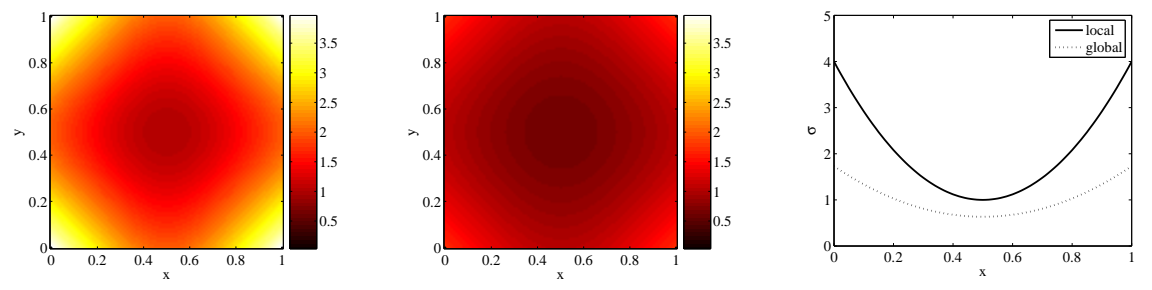

Fig. 1 Map of the standard displacement uncertainty $\sigma_{u}$ (normalized by $\sqrt{2} \sigma p / G_{f} \ell$ ) for local (a) and global (b) approaches. Cuts along the diagonal $x=y$ (c). 


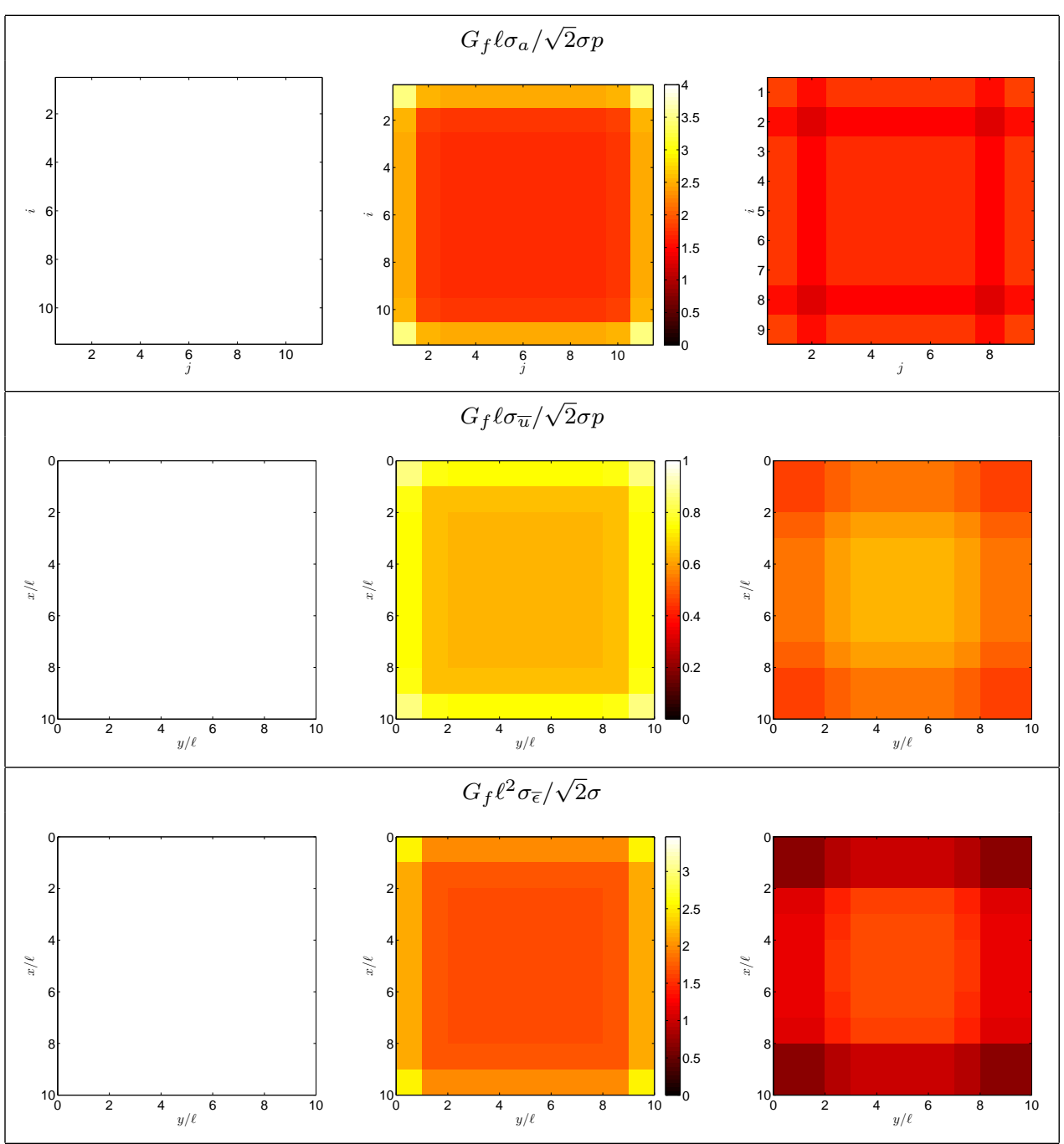

Fig. 2 Standard uncertainties in terms of nodal displacements $\left(\sigma_{a}\right)$, mean displacements per ZOI or element $\left(\sigma_{\bar{u}}\right)$, and mean strains per ZOI or element $\left(\sigma_{\bar{\epsilon}}\right)$ for three different DIC approaches. In the present case, 100 ZOIs are considered for the local approach (left), 100 elements are considered for the global approach (middle), and 64 elements for the global and optimized approach (right). 


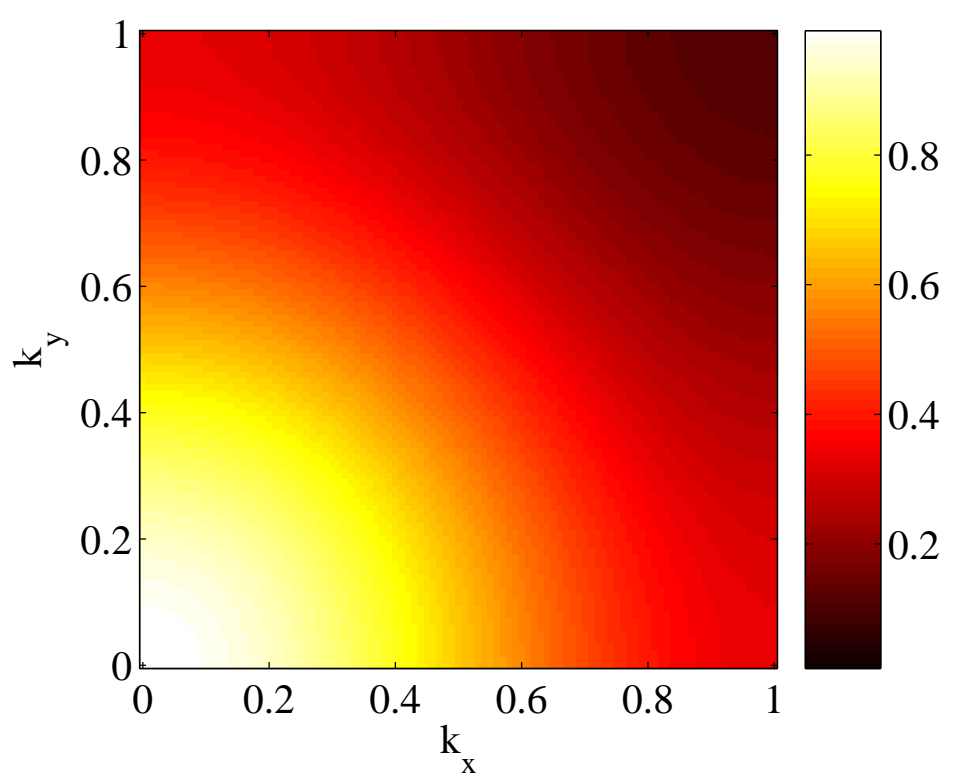

Fig. 3 Map of the eigenvalues in Fourier space. 


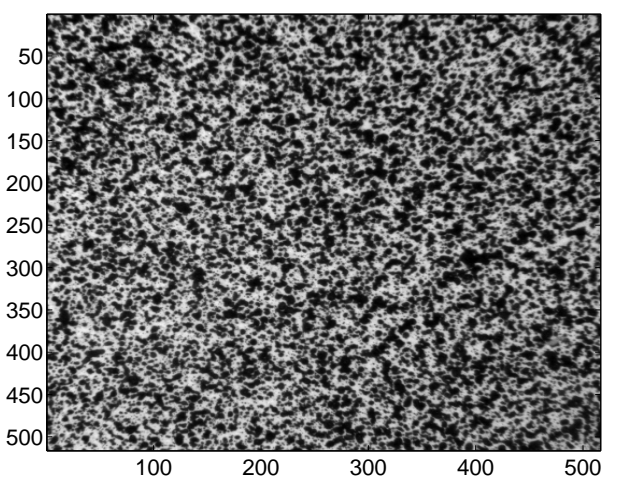

(a)

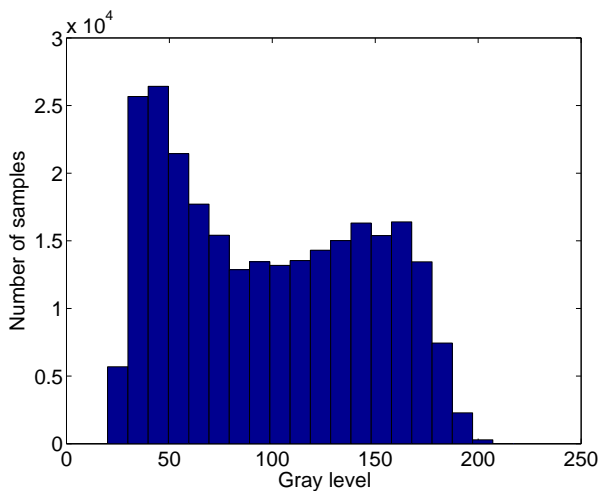

(b)

Fig. 4 Reference picture for the a priori resolution analysis (a), and corresponding histogram (b). The definition of the picture is equal to 256 kpixels with an 8-bit digitization. 


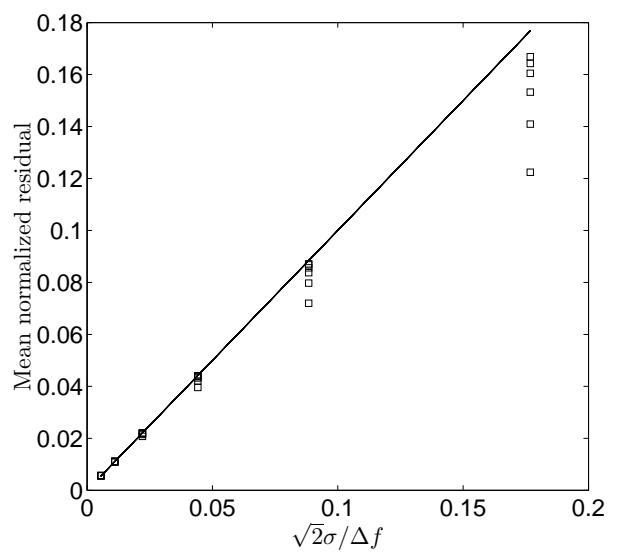

Fig. 5 Normalized RMS residual $\bar{\eta} / \Delta f$ as a function of the dimensionless noise level $\sqrt{2} \sigma / \Delta f$ when $\sigma=1,2,4,8,16,32$ gray levels. The straight line shows a linear interpolation with a slope of 1. 


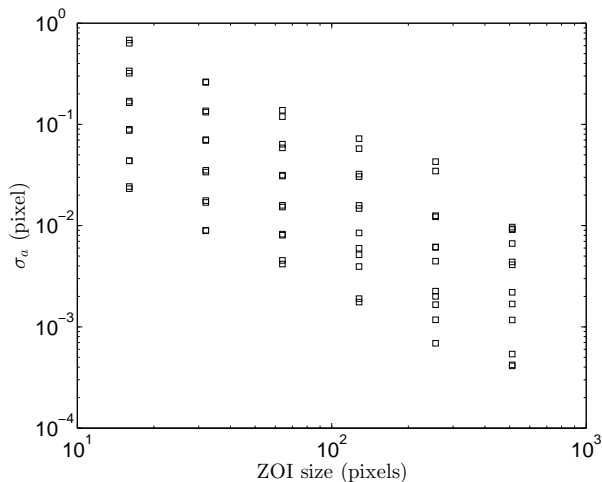

(a)

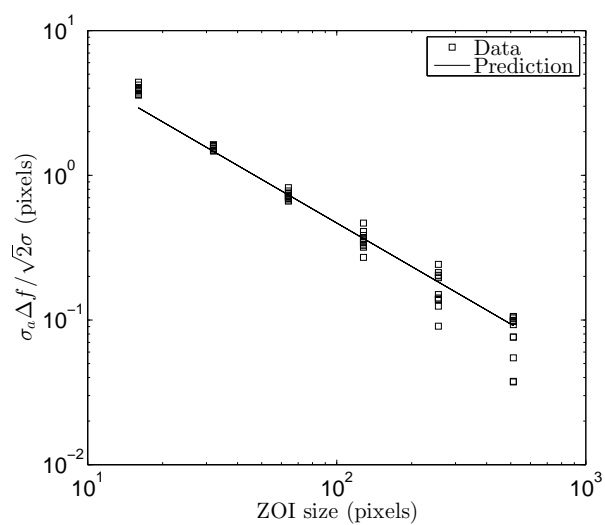

(b)

Fig. 6 Standard displacement uncertainty $\sigma_{a}$ as a function of the ZOI size $\ell$ when $\sigma=$ $1,2,4,8,16,32$ gray levels (a). The higher $\sigma$ the higher $\sigma_{a}$ for a given ZOI size. Normalized standard displacement uncertainty $\sigma_{a} \Delta f / \sqrt{2} \sigma$ as a function of the ZOI size $\ell$. The straight line shows an interpolation by the inverse of the ZOI size. 


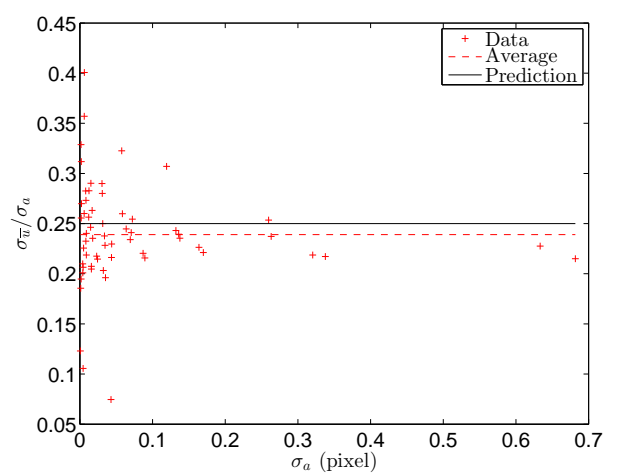

Fig. 7 Normalized standard displacement uncertainty $\sigma_{\bar{u}} / \sigma_{a}$ as a function of $\sigma_{a}$. The solid line shows the result of Equation (21). 


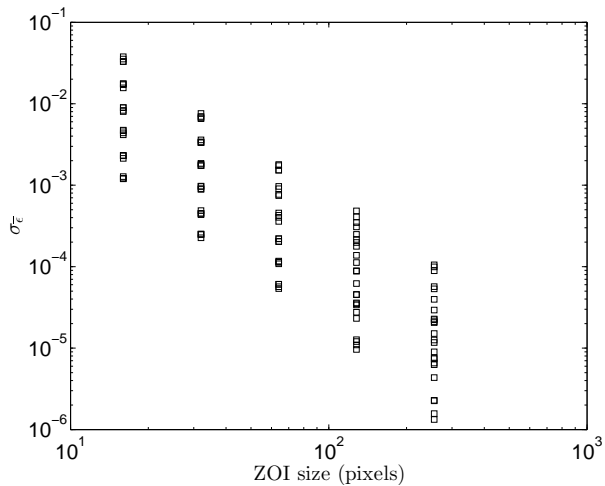

(a)

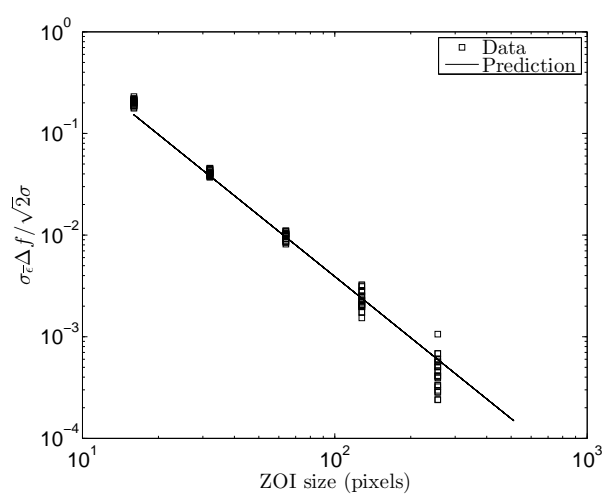

(b)

Fig. 8 Standard strain uncertainty $\sigma_{\bar{\epsilon}}$ as a function of the ZOI size $\ell$ when $\sigma=$ $1,2,4,8,16,32$ gray levels (a). The higher $\sigma$ the higher $\sigma_{\bar{\epsilon}}$ for a given ZOI size. Normalized standard strain uncertainty $\sigma_{\bar{\epsilon}} \Delta f / \sqrt{2} \sigma$ as a function of the ZOI size $\ell$. The solid line shows an interpolation by the inverse of square of the ZOI size. 


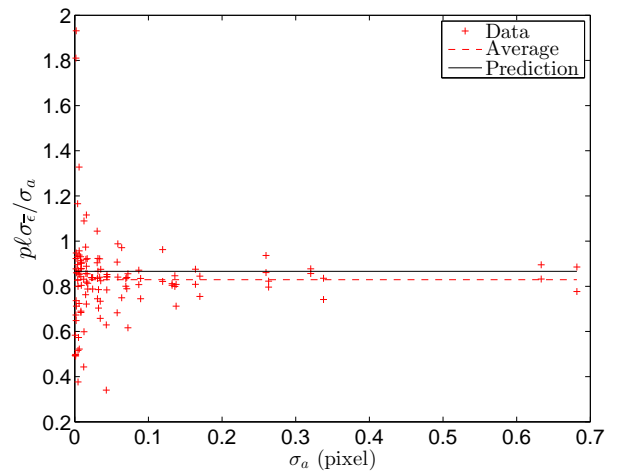

Fig. 9 Normalized standard strain uncertainty $p \ell \sigma_{\bar{\epsilon}} / \sigma_{a}$ as a function of $\sigma_{a}$. The solid line shows the result of Equation (27). 


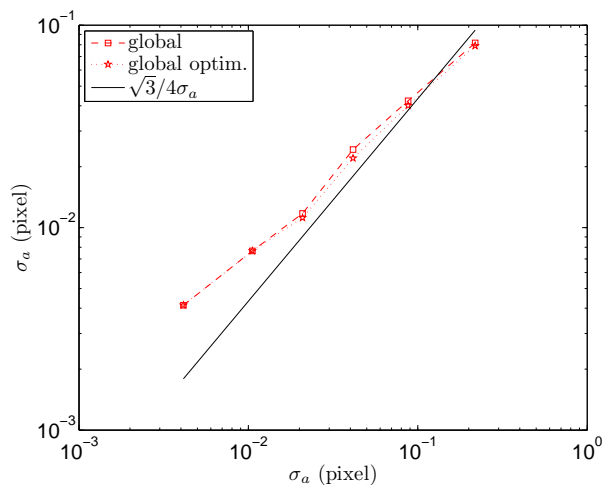

(a)

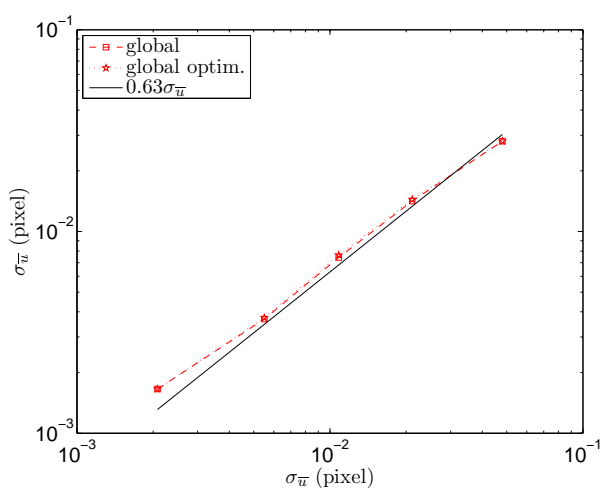

(b)

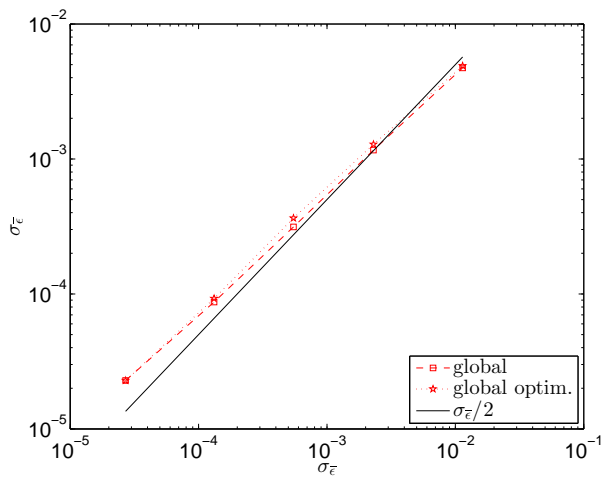

(c)

Fig. 10 Standard displacement uncertainty of the measured degrees of freedom (a), of the mean displacement per ZOI (b), of the mean strain per element for a global and optimized approach as functions of the same quantity for a local approach. 


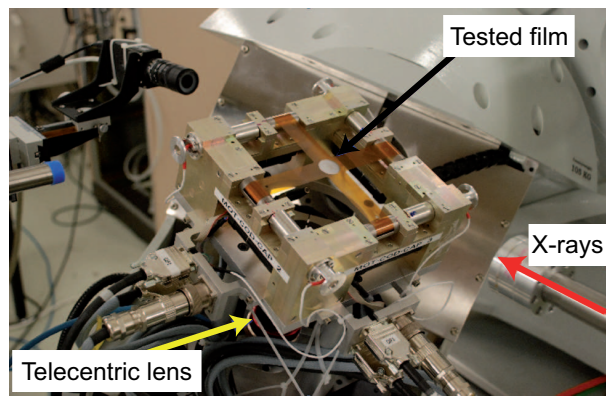

(a)

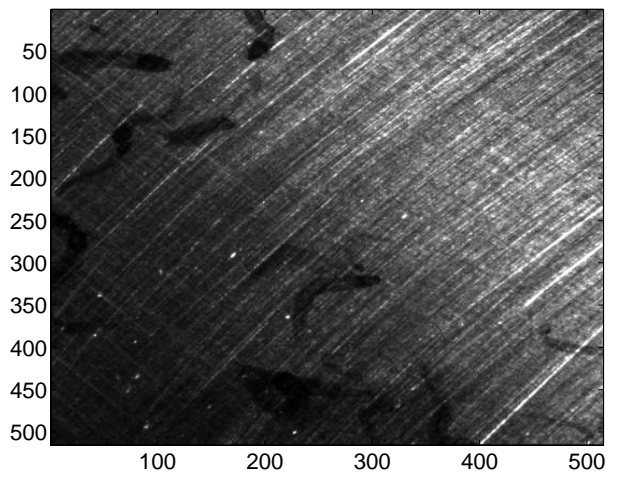

(b)

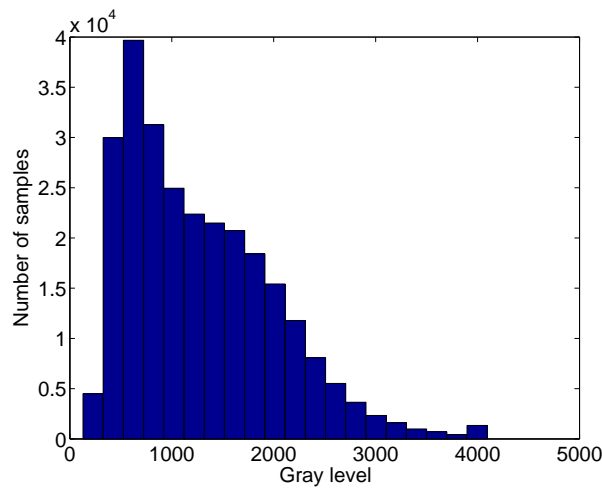

(c)

Fig. 11 Biaxial testing machine (a) in DiffAbs line at SOLEIL synchrotron [44]. Reference picture of a natural texture used in in situ experiments (b), and corresponding histogram (c). The definition of the picture is equal to 256 kpixels with a 12-bit digitization. 


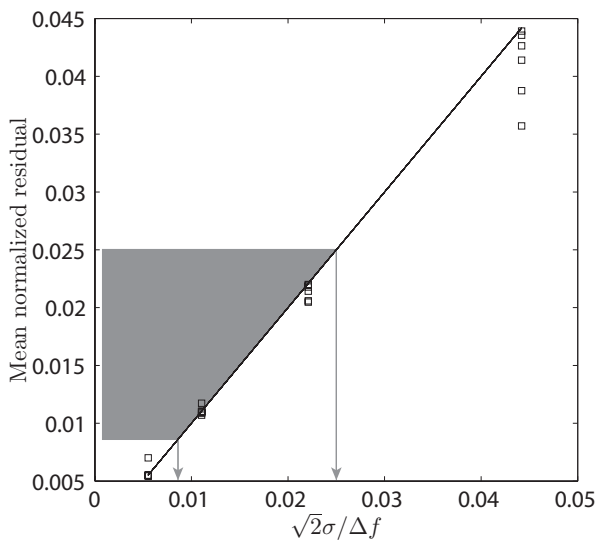

Fig. 12 Normalized RMS residual $\bar{\eta} / \Delta f$ as a function of the dimensionless noise level $\sqrt{2} \sigma / \Delta f$ when $\sigma / \Delta f=1 / 256,1 / 128,1 / 64,1 / 32$. The solid line shows a linear interpolation with a slope of 1 . The shaded area depicts the variation of correlation residuals for the ten analyzed pictures. 


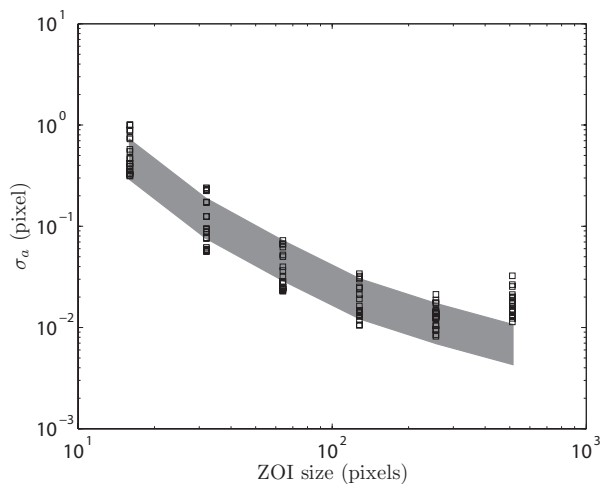

(a)

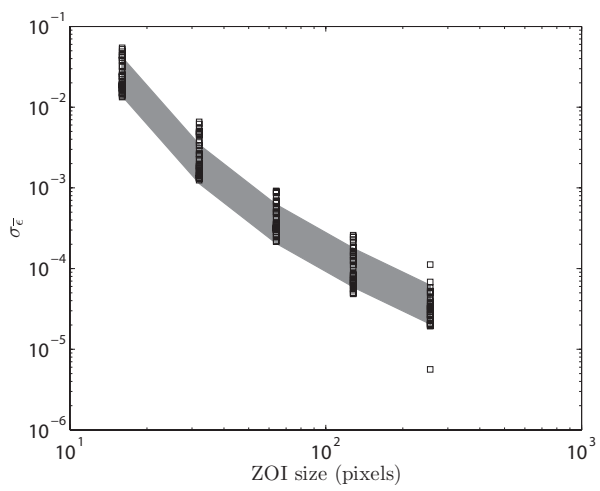

(b)

Fig. 13 Standard displacement uncertainty $\sigma_{a}$ (a) and strain uncertainty $\sigma_{\bar{\epsilon}}$ (b) as functions of the ZOI size $\ell$ for the ten analyzed pictures. The gray shaded areas show the predictions based on the a priori resolution analysis. 


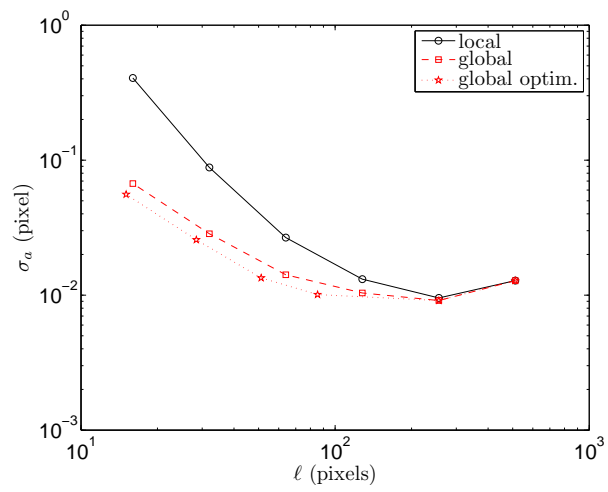

(a)

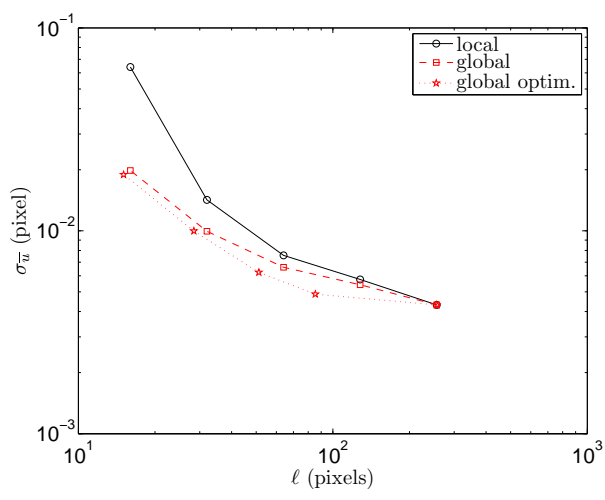

(b)

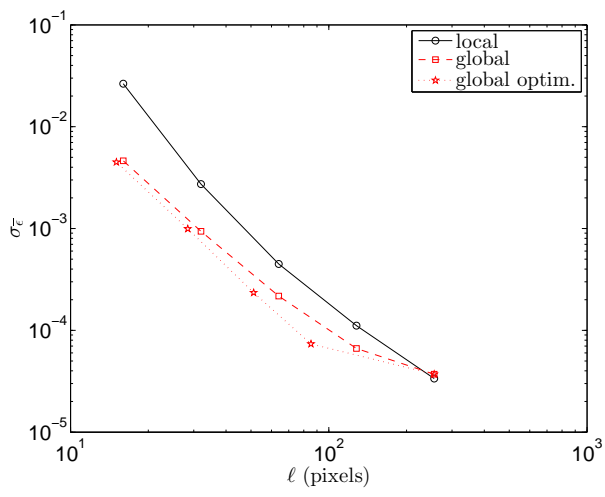

(c)

Fig. 14 Standard displacement uncertainty of the measured degrees of freedom (a), of the mean displacement per ZOI or element (b), of the mean strain per ZOI or element as functions of ZOI or element size $\ell$ for the three approaches used herein. 


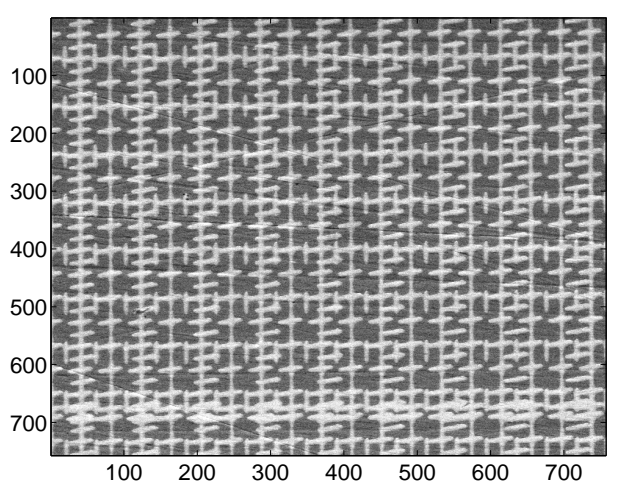

(a)

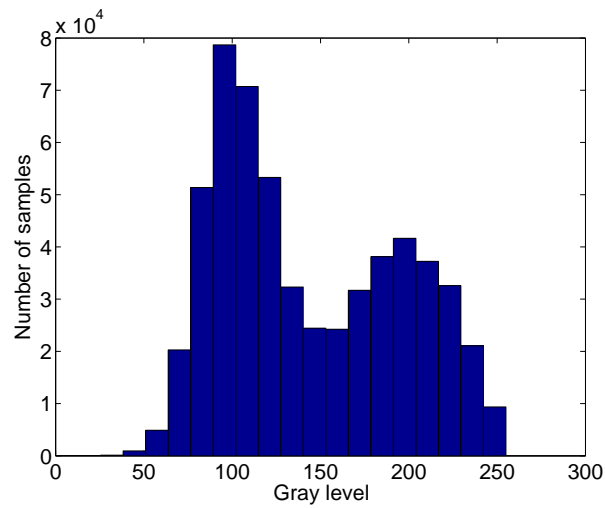

(b)

Fig. 15 Reference SEM picture for which a grid was deposited (a), and corresponding histogram (b). The definition of the picture is equal to 563 kpixels with an 8-bit digitization. 


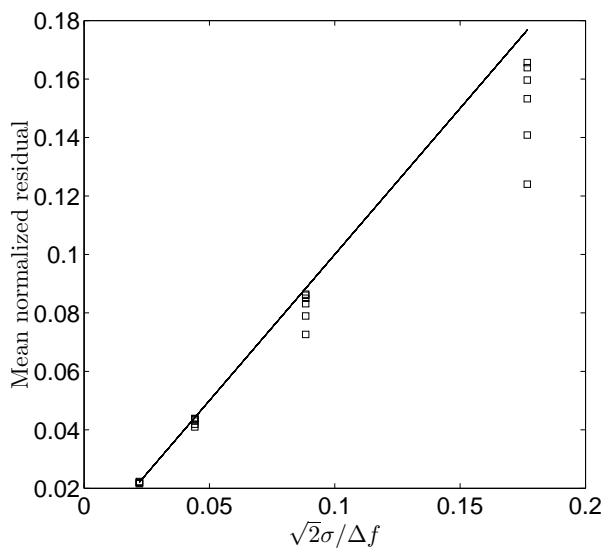

Fig. 16 Normalized RMS residual $\bar{\eta} / \Delta f$ as a function of the dimensionless noise level $\sqrt{2} \sigma / \Delta f$ when $\sigma / \Delta f=1 / 64,1 / 32,1 / 16,1 / 8$. The solid line shows a linear interpolation with a slope of 1. 


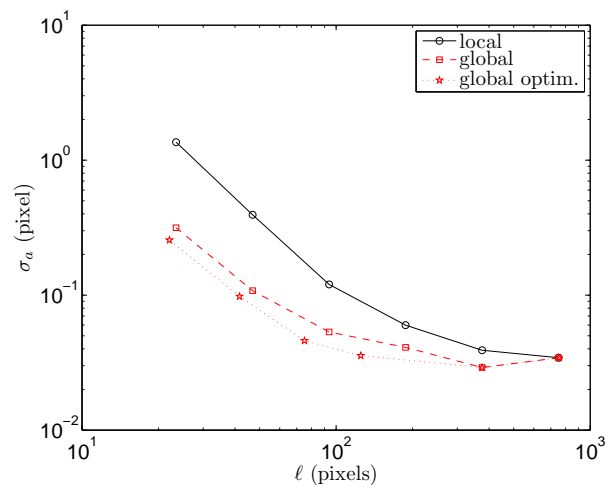

(a)

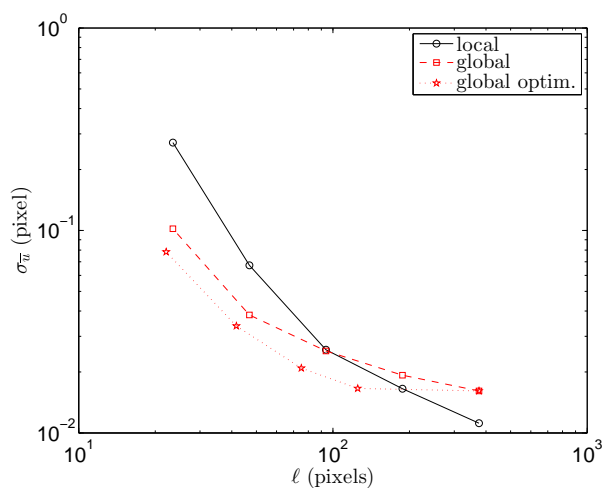

(b)

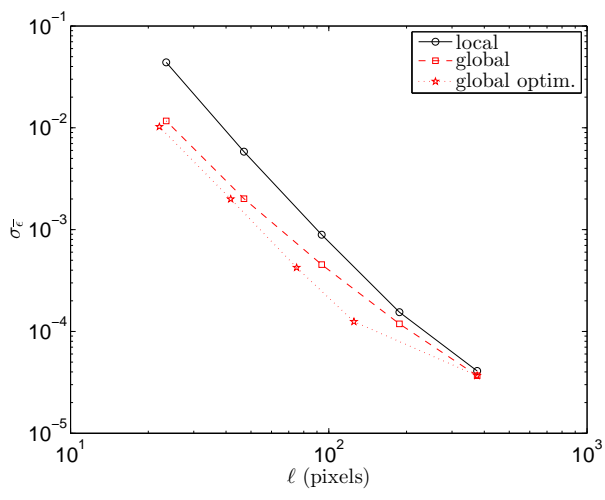

(c)

Fig. 17 Standard displacement uncertainty of the measured degrees of freedom (a), of the mean displacement per ZOI or element (b), of the mean strain per ZOI or element as functions of ZOI or element size $\ell$ for the three approaches used herein. 